\title{
Off-axis holographic laser speckle contrast imaging of blood vessels in tissues
}

\author{
Arkady Abdurashitov \\ Olga Bragina \\ Olga Sindeeva \\ Sindeev Sergey \\ Oxana V. Semyachkina-Glushkovskaya \\ Valery V. Tuchin
}




\title{
Off-axis holographic laser speckle contrast imaging of blood vessels in tissues
}

\author{
Arkady Abdurashitov, ${ }^{\mathrm{a}, \star}$ Olga Bragina, ${ }^{\mathrm{b}, \mathrm{c}}$ Olga Sindeeva, ${ }^{\mathrm{b}, \mathrm{d}, \mathrm{e}}$ Sindeev Sergey, \\ Oxana V. Semyachkina-Glushkovskaya, ${ }^{\mathrm{b}}$ and Valery V. Tuchin ${ }^{\mathrm{a}, \mathrm{f}, \mathrm{g}}$ \\ ${ }^{a}$ Saratov National Research State University, Research-Educational Institute of Optics and Biophotonics, Saratov, Russia \\ bSaratov National Research State University, Department of Human and Animal Physiology, Saratov, Russia \\ 'University of New Mexico School of Medicine, Department of Neurosurgery, Albuquerque, New Mexico, United States \\ ${ }^{\mathrm{d} Q u e e n}$ Mary University of London, School of Engineering and Materials Science, London, United Kingdom \\ eSaratov National Research State University, Remote Controlled Theranostic Systems Laboratory, Saratov, Russia \\ Institute of Precision Mechanics and Control RAS, Laboratory of Laser Diagnostics of Technical and Living Systems, Saratov, Russia \\ ${ }^{9}$ National Research Tomsk State University, Interdisciplinary Laboratory of Biophotonics, Tomsk, Russia
}

\begin{abstract}
Laser speckle contrast imaging (LSCI) has become one of the most common tools for functional imaging in tissues. Incomplete theoretical description and sophisticated interpretation of measurement results are completely sidelined by a low-cost and simple hardware, fastness, consistent results, and repeatability. In addition to the relatively low measuring volume with around $700 \mu \mathrm{m}$ of the probing depth for the visible spectral range of illumination, there is no depth selectivity in conventional LSCI configuration; furthermore, in a case of high NA objective, the actual penetration depth of light in tissues is greater than depth of field (DOF) of an imaging system. Thus, the information about these out-of-focus regions persists in the recorded frames but cannot be retrieved due to intensity-based registration method. We propose a simple modification of LSCI system based on the off-axis holography to introduce after-registration refocusing ability to overcome both depth-selectivity and DOF problems as well as to get the potential possibility of producing a cross-section view of the specimen. $\odot 2017$ Society of Photo-Optical Instrumentation Engineers (SPIE) [DOI: 10.1117/1.JBO.22.9.091514]
\end{abstract}

Keywords: laser speckle contrast; off-axis holography; blood flow; depth of field; cross section; numerical refocusing Paper 160827SSR received Dec. 31, 2016; accepted for publication Mar. 27, 2017; published online Apr. 25, 2017.

\section{Introduction}

Noninvasive optical techniques based on dynamic light scattering offer rich possibilities for diagnosing a broad range of diseases. ${ }^{1-3}$ While Doppler methods are relied on for detecting a shift in the carrier frequency of scattered photons, ${ }^{4}$ specklebased techniques, in general, utilized an analysis of time-variant speckle pattern, ${ }^{5}$ produced by random interference phenomenon.

Laser speckle contrast analysis/imaging (LSCA, LSCI) is a promising tool for nondestructive monitoring blood and lymph flows in tissues. ${ }^{6}$ Compare to Doppler-related methods, LSCI provides full-filed flow maps in real time without any scanning hardware, its angle-independent, digital processing is partially done by exposure time of the camera, very low-cost ${ }^{7}$ and robust. However, due to a complex theoretical description of biospeckle formation and its strong dependence on experimental conditions,,${ }^{8,9}$ causing sophisticated interpretation of measuring quantity, ${ }^{10}$ the vast majority of LSCI techniques are rather qualitative than quantitative, unless LSCI is used with another measuring modality to overcome some of these problems. ${ }^{11}$ In addition to that, LSCI has a relatively low measuring volume with around $700 \mu \mathrm{m}$ of the probing depth for the visible spectral range of illumination ${ }^{12}$ and there is no depth selectivity and the possibility to correct errors, related to focus missing, after image registration. Ringuette et al. ${ }^{13,14}$ did an impressive work for real-time missing-focus detection and corresponding correction; however, this technique requires a high-cost spatial light modulator and iterative calculations.

*Address all correspondence to: Arkady Abdurashitov, E-mail: abdurashitovoptics@mail.ru
In this study, we attempted to partially solve the depth-selectivity problem and account for related errors in LSCI method by merging laser speckle contrast and holography to get access to both intensity and phase information, along with ability to correct numerically propagation of the wavefront, i.e., numerical focusing. ${ }^{15}$

\section{Methods}

\subsection{Holography Basics}

To produce a hologram, we have to introduce a reference wave in conventional LSCI configuration. The easiest way to do it is to physically "sandwich" cube beam splitter (BS) between camera body and the objective lens, as shown in Fig. 1 .

As a result of coherent summation of reference and object waves, interference fringes are produced [Fig. 2(a)].

Angle $\theta$ between these waves is needed to provide a separation between orders in spatial spectrum of the hologram presented in Fig. 2(b), that is why this recording scheme is called "off-axis." 15,16

Mathematically, the expression of this coherent summation is (spatial coordinates are hidden for convenience $)^{15}$

$E=\left(R^{2}+O^{2}\right)+R^{*} O+R O^{*}$,

where $E$ is the resulting electric field distribution across the senor, $\left(R^{2}+O^{2}\right)$ is the autocorrelation term, $R^{*} O$ and $R O^{*}$ are the real

1083-3668/2017/\$25.00 @ 2017 SPIE 


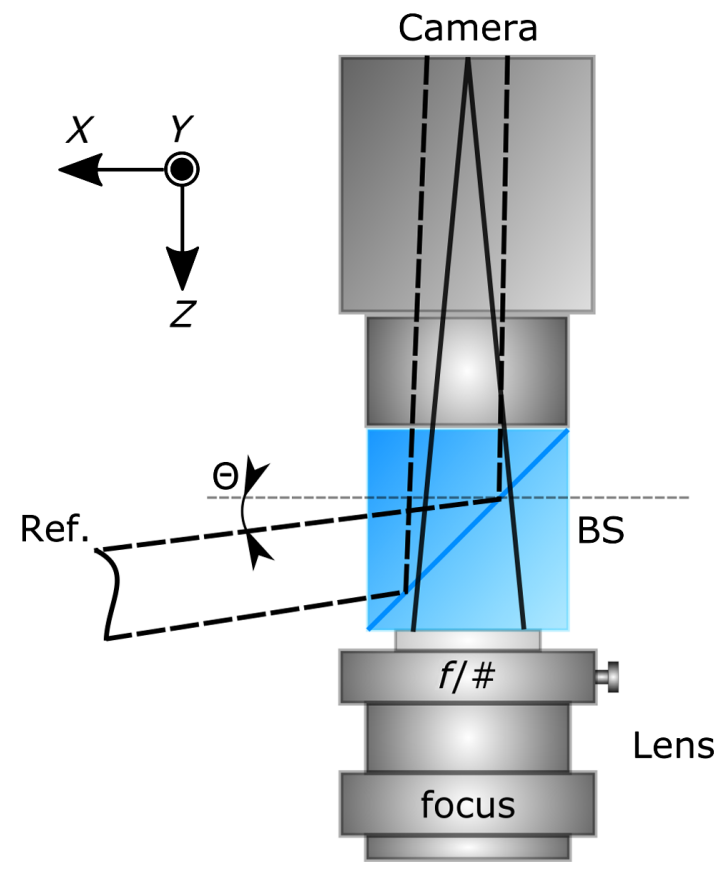

Fig. 1 Insertion of the BS provides ability to introduce a reference wave. On the other hand, it acts like an extension ring in photography, increasing the magnification of optical system by the price of aberrations and internal reflections. Due to holographic nature, these drawbacks can be eliminated numerically by digital processing of the image. $\theta$ is the off-axis angle between reference wave and optical axis of the system.

and twin terms, respectively, $R$ and $O$ are the reference and object waves, respectively. All these components are presented in the spatial spectrum of the hologram shown in Fig. 2(b).

In this study, to reconstruct the real image from the hologram, the angular spectrum approach was used. ${ }^{17}$ After Fourier transform of hologram presented in Fig. 2(a), spatial spectrum presented in Fig. 2(b) is produced. Physically, this means that we decomposed our hologram into elementary plane waves $e^{i k z}$, propagating along $z$-direction at different angles with respect to it. In our notation, $z$-direction means the indepth coordinate of the object. The angle is determining the value of the spatial frequency. After this step, a spatial filtering in Fourier domain is done by cutting the real-image term from the frequency spectrum. Padding this cut term with zeroes has to be done to preserve resolution of the reconstructed image. After padding, to simulate propagation of the wavefront, pointwise multiplication of padded array and the transfer function or "propagator" is done. The equation for transfer function is ${ }^{17}$

$\operatorname{transfer}(z)=\exp \left(-i k z \sqrt{1-\lambda^{2} f_{x}^{2}-\lambda^{2} f_{y}^{2}}\right)$

where $k=2 \pi / \lambda$ is the wavenumber, $z$ is the in-depth coordinate, i.e., how long wavefront should propagate, $\lambda$ is the wavelength of illumination, $f_{x}$ and $f_{y}$ are the corresponding spatial frequencies along $x$ - and $y$-directions. Graphical representation of transfer function is shown in Fig. 3.

Completed mathematical notation to reconstruct wavefront at an arbitrary distance $z$ from the initial recording plane is as follows:

$U(x, y, z)=\mathrm{FFT}^{-1}[\mathrm{FFT}($ holo $) \operatorname{transfer}(z)]$,

where FFT denotes the fast Fourier transform, power -1 is the inverse operator, holo is the recorded hologram, and $U(x, y, z)$ is the reconstructed wavefront (in a complex representation) at position $z$.

To validate numerical focusing capability of our setup, intentionally, unfocused image of $\mathrm{F}$ letter was recorded and sharply focused numerically after the registration (Fig. 4).

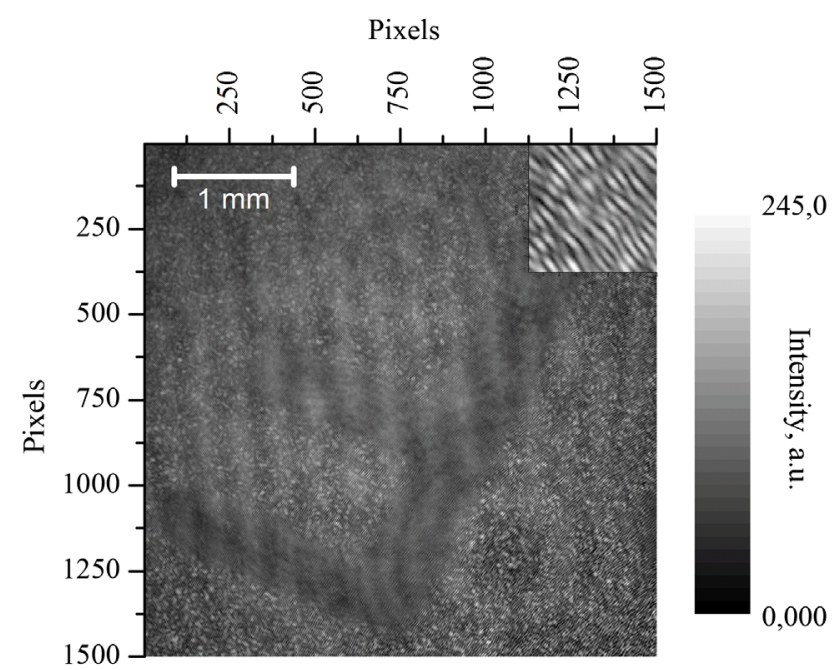

(a)

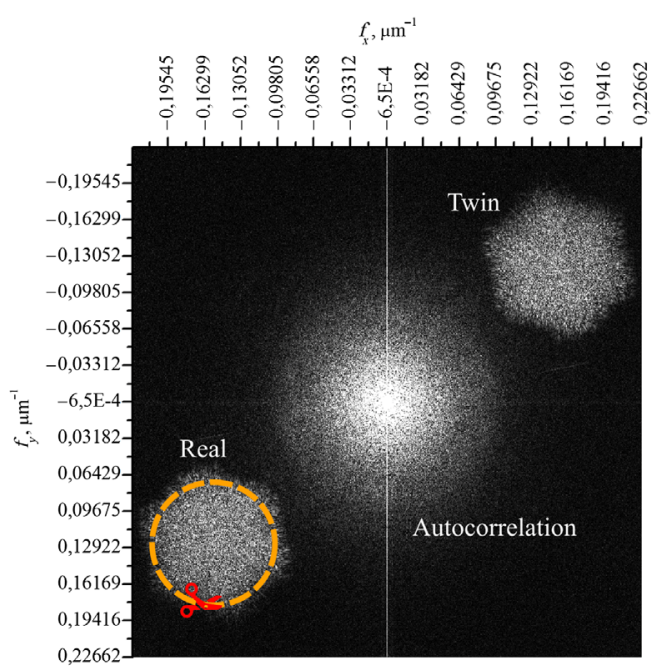

(b)

Fig. 2 (a) Typical off-axis hologram. Fringes are orientated close to 45 deg to provide maximum room for separation in frequency space between interference orders. Interference pattern is curved because of speckles. In this work, unlike the vast majority, we preserve speckles to use them in $\mathrm{LSCl}$ after reconstruction. (b) Spatial spectrum is obtained by performing FFT of the recorded hologram. As predicted by Eq. (1), there are three terms in this image marked as "real," "autocorrelation," and "twin." Off-axis configuration with right adjusted angle $\theta$ (physically, its fringe period $T=\lambda / \theta$ ) provides needed separation between interference orders. 


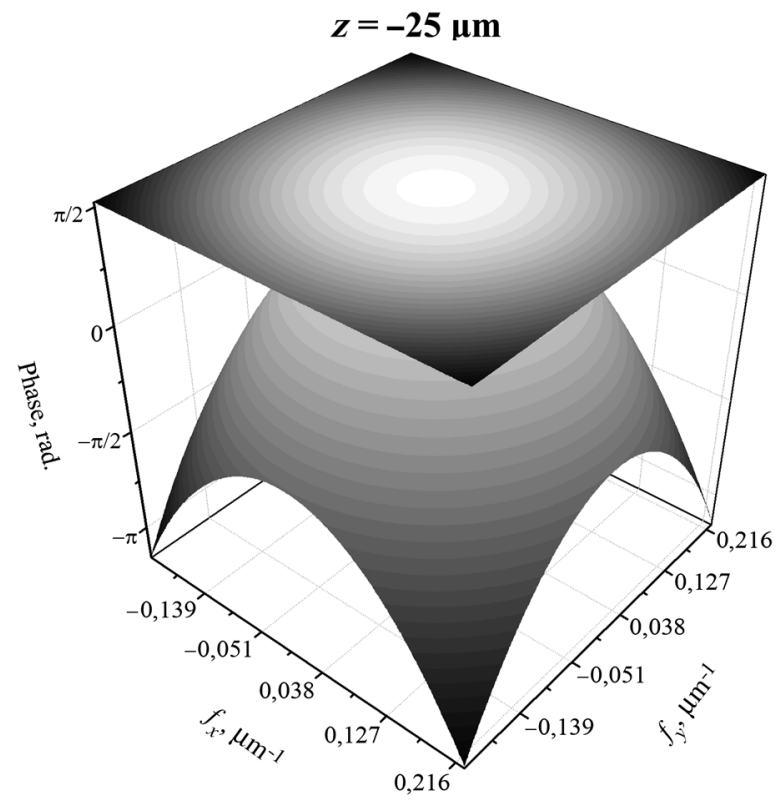

(a)

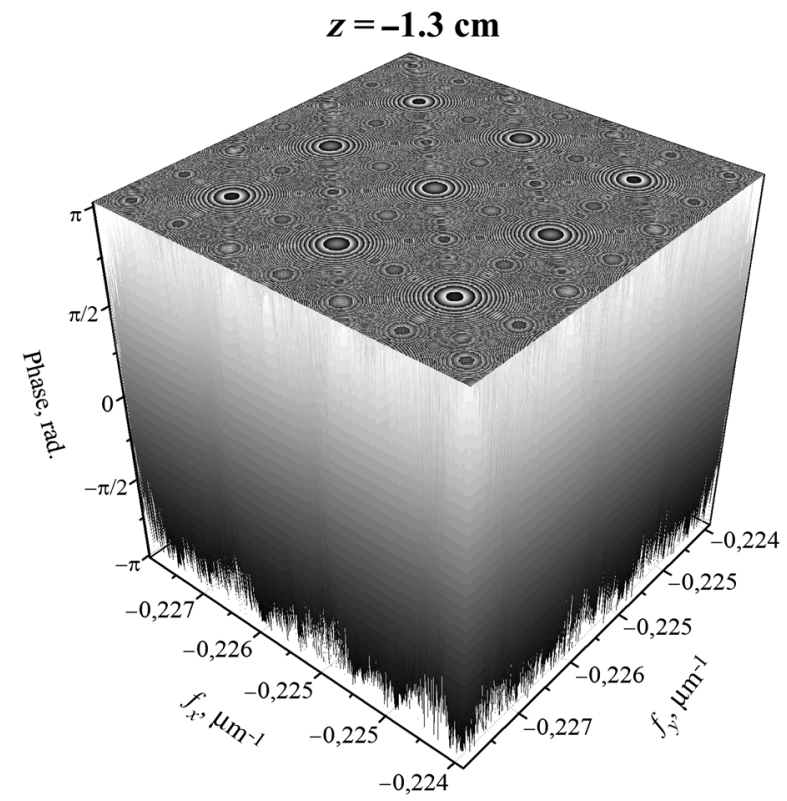

(b)

Fig. 3 Graphical representation of transfer function [Eq. (2)] for two different values of $z$-direction. This function shows how phase relations of elementary wavelets with particular set of frequencies $f_{x}$ and $f_{y}$ change during propagation. To compute this figure, following setting was used $\lambda=633 \mathrm{~nm}$, (a) $z=-25 \mu \mathrm{m}$ and (b) $z=-1.3 \mathrm{~cm}$. In (b), phase values are wrapped by a modulo $\pi$.

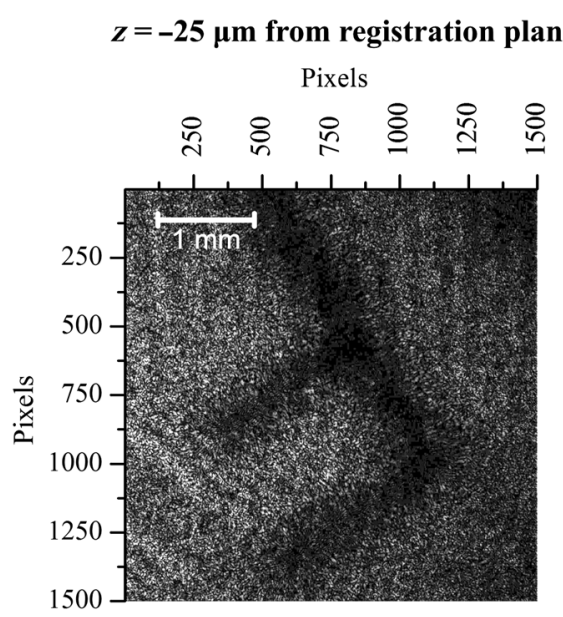

(a)

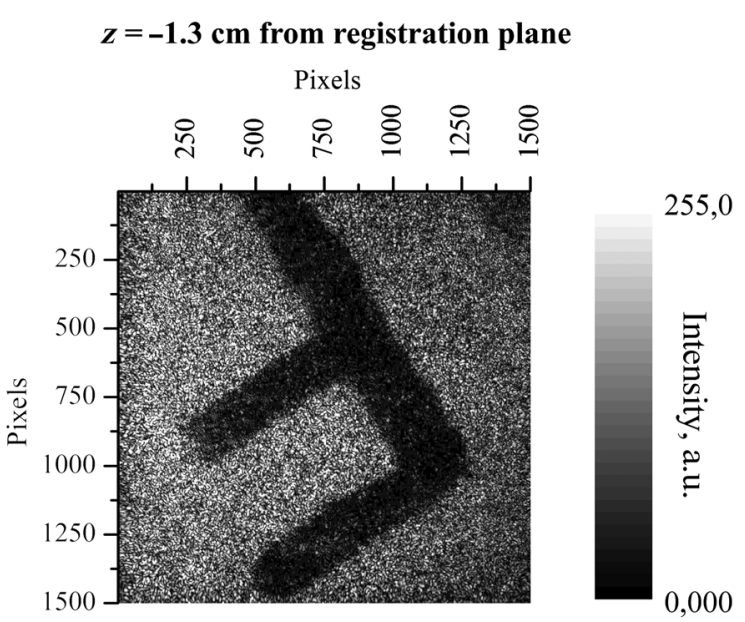

(b)

Fig. 4 Effect of numerical focusing. Initially recorded unfocused image of $F$ letter reconstructed using different propagation distances. As shown in (a) and (b), angular spectrum approach is working for a large range of propagation distances.

\subsection{Laser Speckle Contrast Imaging/Analysis}

To analyze flow dynamics in tissue, time-domain $\operatorname{LSCA}^{18}$ was applied to reconstruct images. Time-domain selection was dictated by holography requirements: speckles should not be blurred during exposure time, otherwise interference fringes will disappear with the ability to separate interference orders in the spatial spectrum. To characterized flow, temporal speckle contrast was calculated using the following equation:

$K_{t}=\frac{\sigma_{t}}{\langle I\rangle_{t}}$

where $K_{t}$ is the temporal speckle contrast, $\sigma_{t}$ and $\langle I\rangle_{t}$ are the standard deviation of intensity fluctuations in a single pixel and the mean value of intensity for the same pixel over time $t$, respectively (For the time-domain, speckle contrast equal to zero is corresponding to no flow. That for the proposed method means no intensity fluctuations for a given pixel from one frame to the other).

\section{Experimental Protocol}

\subsection{Optical Arrangement}

Optical setup was constructed according to the arrangement shown in Fig. 1. Complementary metal-oxide-semiconductor Basler Aca2500-14 gm (pixel size $2.2 \mu \mathrm{m}$ ) was used as a detector. Object was illuminated with single-mode HeNe laser 
Thorlabs HNL210L, $632.8 \mathrm{~nm}, 21 \mathrm{~mW}$, linearly polarized. To split laser irradiation into "object" and "reference" parts, singlemode polarization-maintained fiber splitter Thorlabs PMC63050B-APC 50:50 was utilized. Tamron 21HC, $50 \mathrm{~mm}$ was used as an imaging lens. $F$-stop of objective was set to $f / 6$, but due to extension length provided by $\mathrm{BS}$, the effective $F$-stop was calculated according to the following equation:

$F-$ stop $_{\text {eff }}=F-$ stop $\times(1+$ Magnification $)$.

Considering magnification of the system (0.85), effective $F$-stop is $\sim 11$. Speckle size was calculated accordingly ${ }^{1}$

$d_{\text {speckle }}=2.44 \lambda(1+$ Magnification $) F-$ stop $_{\text {eff }}$,

and was $\approx 30 \mu \mathrm{m}$ With this, pixel/speckle ratio is $\sim 15$ and we are comfortably above Nyquist criteria. ${ }^{19}$ Illumination angle was set to $45 \mathrm{deg}$. Angle theta was set to $\sim 5 \mathrm{deg}$ to satisfy Nyquist criteria (more than 2 pixels per one fringe period) and thus to optimize the distance between orders in the spatial spectrum. Optical path difference between object and reference waves was set to zero. Exposure time of the sensor was equal to $5 \mathrm{~ms}$, and frame rate of the recording was set to 40 frames per second. Normally, blood flow in animals is high, especially for brain. ${ }^{20}$ However, due to fact that animal under study was affected by acetylcholine, blood flow was reduced sufficiently to minimize speckle blurring at 5-ms exposure time. Signal-tonoise ratio (SNR) of this setup mainly depends on experimental conditions, such as motion artifacts of the object and phase stability of the interferometer. In this case, fringe contrast is the numeric value for SNR. In our study, fringe contrast was $\sim 0.6$, which is a reasonable value for tissues.

\subsection{Animal Protocol}

Experiments were performed on newborn mongrel rats, 10-days old. All procedures were done in accordance with the Guide for the Care and Use of Laboratory Animals. ${ }^{21}$ Surgery was performed under general anesthesia with Hypnorm $(0.4 \mathrm{ml} / \mathrm{kg}$, Janssen Saunderton, Buckinghamshire, England) diluted in sterile water (Gothelf et al.). ${ }^{22}$ The anesthesia was administered as intraperitoneal injections. Anesthetic depth was assessed by periodically monitoring the rear foot reflex.

The relative cerebral blood flow measurements were carried out in immobilized anesthetized rats. For improvement of image quality of blood flow in vessels of the brain in neonatal rats, parietal bones were partially removed; however, the "dura matter" was left intact. To stimulate parasympathic nervous system activity along with blood flow reduction, the acetylcholine chloride $(0.3 \mu \mathrm{g} / \mathrm{kg}$, Sigma-Aldrich, St. Louis, Missouri) was injected through a polyethylene catheter $(0.28 \mathrm{~mm} \mathrm{ID,}$ $0.61 \mathrm{~mm}$ OD, Portex, Smiths Medical International Ltd., UK), which was implanted into the jugular vein. The drug was administered through the catheter to prevent the slightest movement of an object, which is typical in normal drug administration through the tail vein.

\section{Results}

As described in Secs. 2 and 3, protocols were applied as proofof-concept approach to investigate blood flow dynamics in rat brain cortex. Each off-axis hologram was digitally processed using home-made software based on NI LabView and NI Vision to simulate propagation of wavefront in a range from $-3 \mathrm{~mm}$ to $3 \mathrm{~mm}$ from the initial registration plane. Two hundred numerically calculated raw speckle frames were used to obtain one temporal LSCI image.

It is clearly seen in Fig. 5 that at initial focus position, small capillaries on both sides from large vessel are defocused and we could not provide reliable measuring of dynamic properties, such as speckle contrast and diameter. If we now shift the registration plane by $-900 \mu \mathrm{m}$, these capillaries move into sharp focus region and become available for measuring. Such modification of a conventional LSCI technique gives a right to make a small mistake in focusing of the optical system used, because these errors can be corrected numerically after image registration.

Cross-section view can be achieved by taking a slice across the line of interest (purple line in Fig. 5) through images, which were reconstructed with monotonically changed $z$-parameter.

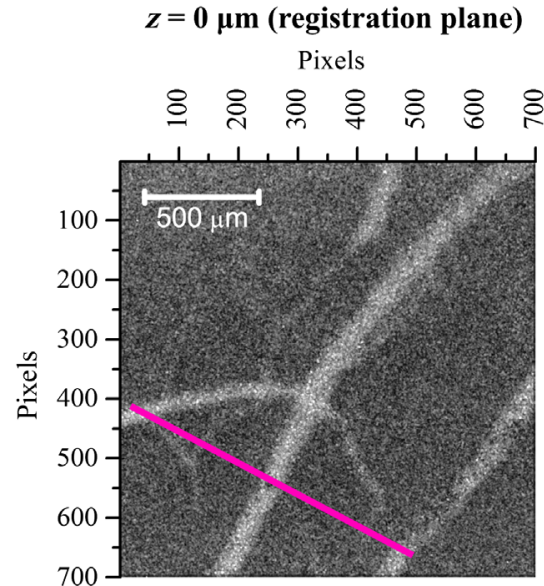

(a)

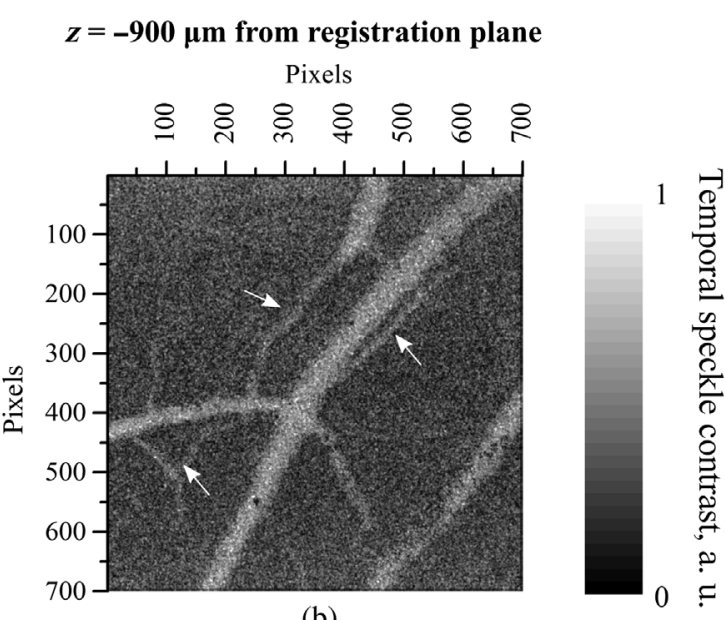

(b)

Fig. $5 \mathrm{LSCl}$ images calculated from reconstructed frames. At the registration plane, (a) small vessels are in mis-focus position and unavailable for measuring. To compensate for this, holography nature of the recording allows us to move numerically the registration plane on $-900 \mu \mathrm{m}$ (b) to provide sharp focusing of these small vessels. 


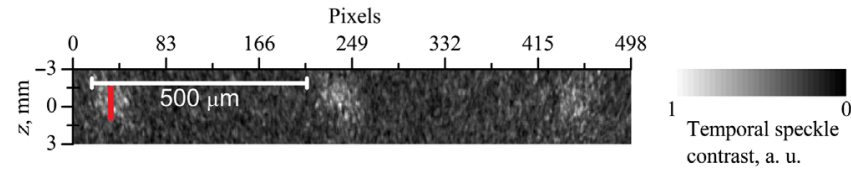

Fig. 6 Temporal speckle contrast distribution across purple line of Fig. 5 with monotonically changed $z$-parameter. It is a cross-sectional view of the specimen in some point of view. However, more detailed interpretation and advance digital processing are needed to compensate for unnatural stretching of vessels in $z$-direction.

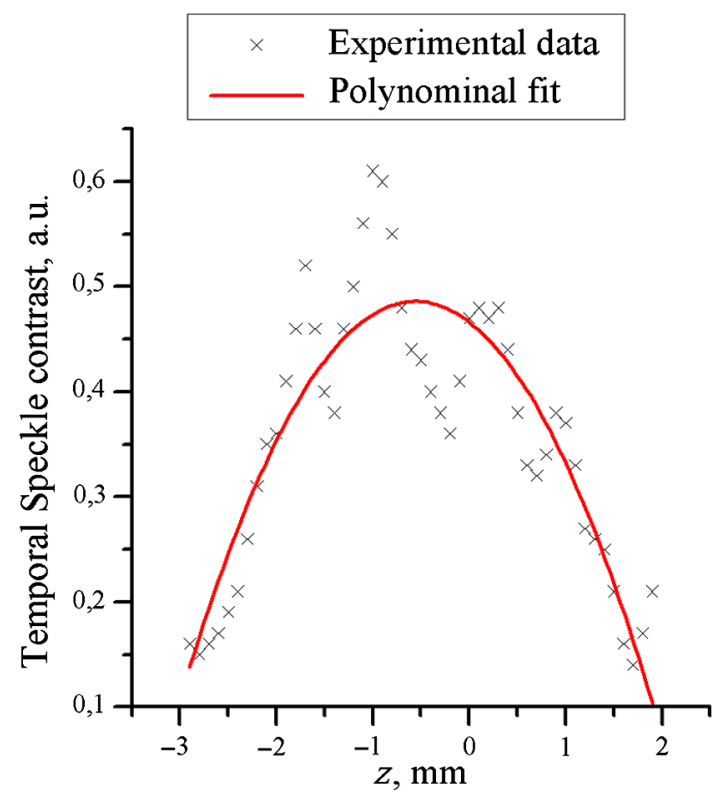

Fig. 7 Depth profile of a single vessel. Polynomial fit shows good agreement with expected parabolic distribution of the speckle contrast across the vessel.

We intentionally did not call $z$-parameter as a depth because after numerical propagation of wavefront we obtained not the intensity distribution at a certain depth in the object, but a superposition of in-focus and out-of-focus parts of object image recorded in an arbitrary position $z$. Figure 6 shows such crosssectional view.

Three bright spots correspond to three different vessels. Depth profile of a single vessel marked by the red line is demonstrated in Fig. 7. Polynomial fit shows good correlation with parabolic distribution across the vessel.

It is clearly seen from these images that vessels appear to be somehow stretched in $z$-direction. More detailed explanation of this phenomenon will be discussed in Sec. 5 .

\section{Discussion and Conclusion}

Holography implies few strict restrictions on optical setup, which makes this modification a bit more expensive, compared to conventional setup. An off-axis scheme requires enough temporal coherence of the source. Integration time of the sensor should be reduced and frame rate of the recording should be increased to eliminate spatial speckle blurring. As a result, overall laser power needs to be increased, which is unwanted in some cases. Transistor-transistor logic-modulated diode laser can solve problems associated with the needed small exposure time and high power. In this case, the exposure time of the sensor can be fixed and the laser pulse duration can be adjusted to the desired optimal value. Temporal speckle contrast analysis is more suitable for such configuration; however, the reconstructed frames can be digitally averaged to obtain spatial speckle blurring and then processed using spatial speckle contrast analysis.

The proposed device is different from a common-path interferometry, it is robust in getting of the phase information and susceptible to vibrations. The phase information obtained from the reconstructed images is the phase fluctuations caused by moving erythrocytes and averaged over exposure time.

In the cross-sectional view, there are a few problems needed to be overcome as well. One of them is depth of field (DOF) of the optical system. If we are trying to propagate wavefront within DOF volume, we barely see the difference between adjacent frames. Thus, DOF needs to be reduced to increase the sensitivity of refocusing. Longitudinal speckle size is the second big problem. In general, subjective speckles are stretched in the propagation direction $z$. Typical longitudinal speckle size is a few tens of microns, and it is strongly limiting depth resolution of resulting cross-sectional view. In addition to that, there is an overlapping of information between adjacent frames. On the same image, there are in-focus and out-of-focus parts, and this makes the cross-sectional view to be additionally stretched in $z$-direction. Deconvolution microscopy ${ }^{23}$ can solve this problem, but precise measure of the point spread function is needed.

From above, we can conclude that by introducing minimum number of optical elements in conventional LSCI setup we can greatly increase its measuring capability with the ability to correct mis-focus errors after the registration process and obtain both phase and intensity information and cross-sectional view of the object. However, careful digital processing and interpretation of the results are needed to obtain true depth-resolved slicing.

\section{Disclosures}

No conflicts of interest, financial or otherwise, are declared by the authors.

\section{Acknowledgments}

We would like to acknowledge associate professor Anton Grebenuyk and $\mathrm{PhD}$ student Oleg Grishin for practical advising in holography. This work was supported by Grant of Russian Science Foundation No. (16-15-10252). V.V.T. was supported by grant of the President of the Russian Federation NSh7898.2016.2.

\section{References}

1. D. A. Boas and A. K. Dunn, "Laser speckle contrast imaging in biomedical optics," J. Biomed. Opt. 15(1), 011109 (2010).

2. V. V. Tuchin Ed., Handbook of Optical Biomedical Diagnostics. LightTissue Interaction, Volume 1, Methods, Vol. 2, 2nd ed., SPIE Press, Bellingham, Washington (2016).

3. J. Men et al., "Optical coherence tomography for brain imaging and developmental biology," IEEE J. Sel. Top. Quantum Electron. 22, 1-13 (2016).

4. A. F. Fercher et al., "Optical coherence tomography-principles and applications," Rep. Prog. Phys. 66(2), 239-303 (2003).

5. A. F. Fercher and J. D. Briers, "Flow visualization by means of singleexposure speckle photography," Opt. Commun. 37(5), 326-330 (1981).

6. I. V. Fedosov and V. V. Tuchin, "Bioflow measuring: laser Doppler and speckle techniques," in Handbook Coherent-Domain Optical Methods: 
Biomedical Diagnostics, Environmental Monitoring and Material Science, pp. 487-563, Springer, New York (2013).

7. L. M. Richards et al., "Low-cost laser speckle contrast imaging of blood flow using a webcam," Biomed. Opt. Express 4(10), 2269-2283 (2013).

8. D. Briers et al., "Laser speckle contrast imaging: theoretical and practical limitations," J. Biomed. Opt. 18(6), 066018 (2013).

9. K. Khaksari and S. J. Kirkpatrick, "Combined effects of scattering and absorption on laser speckle contrast imaging," J. Biomed. Opt. 21(7), 076002 (2016).

10. S. M. S. Kazmi et al., "Flux or speed? Examining speckle contrast imaging of vascular flows," Biomed. Opt. Express 6(7), 2588-2608 (2015).

11. A. Nadort et al., "Quantitative blood flow velocity imaging using laser speckle flowmetry," Sci. Rep. 6, 25258 (2016).

12. M. A. Davis, S. M. S. Kazmi, and A. K. Dunn, "Imaging depth and multiple scattering in laser speckle contrast imaging," J. Biomed. Opt. 19(8), 086001 (2014).

13. D. Ringuette et al., "Reducing misfocus-related motion artefacts in laser speckle contrast imaging," Biomed. Opt. Express 6(1), 266-276 (2015).

14. D. Ringuette, I. Sigal, and O. Levi, "Robust estimation of vessel misfocus and real-time misfocus correction in laser speckle contrast imaging," Proc. SPIE 9333, 933302 (2015).

15. N. Verrier and M. Atlan, "Off-axis digital hologram reconstruction: some practical considerations," Appl. Opt. 50(34), H136-H146 (2011).

16. E. N. Leith and J. Upatnieks, "Reconstructed wavefronts and communication theory," J. Opt. Soc. Am. 52(10), 1123 (1962).

17. J. W. Goodman, "Introduction to Fourier optics," 3rd Ed., in Optical Engineering p. 491 (2004).

18. P. Li et al., "Imaging cerebral blood flow through the intact rat skull with temporal laser speckle imaging," Opt. Lett. 31(12), 1824-1826 (2006).

19. S. J. Kirkpatrick, D. D. Duncan, and E. M. Wells-Gray, "Detrimental effects of speckle-pixel size matching in laser speckle contrast imaging," Opt. Lett. 33(24), 2886-2888 (2008).

20. S. Yuan et al., "Determination of optimal exposure time for imaging of blood flow changes with laser speckle contrast imaging," Appl. Opt. 44(10), 1823-1830 (2005).

21. J. C. Garber et al., "Guide for the care and use of laboratory animals," 8th ed., National Academies Press, Washington (2011).

22. A. Gothelf, P. Hojman, and J. Gehl, "Change in hemoglobin levels due to anesthesia in mice: an important confounder in studies on hematopoietic drugs," Biol. Proced. Online 11(1), 325 (2009).

23. J. B. Sibarita, "Deconvolution microscopy," Adv. Biochem. Eng./ Biotechnol. 95, 201-243 (2005).
Arkady Abdurashitov is a PhD student in the Department of Optics and Biophotonics at Saratov National Research State University. His research interests include biomedical imaging, laser speckle contrast imaging, optical coherence tomography, data processing, and visualization. He successfully attended a Biophotonics and Imaging Graduate Summer School in Ireland.

Olga Bragina is a PhD student at the Department of Human and Animal Physiology at Saratov National Research State University. Her research interests include brain stimulation, brain pathology, histology, neurobehavioral, biomedical imaging, and laser speckle contrast imaging. She is a member of the Society for Neuroscience.

Olga Sindeeva works at the Remote Controlled Theranostic Systems Lab achieved at Saratov State University. She achieved a PhD in physiology in July 2016. Her research interests belongs to the medical applications of nanoparticles, vessel's and circulatory physiology, and experimental surgery. She was awarded by Scholarship of the Russian Federation Government, Scholarship of the President of Russian Federation, and Scholarship of the President of Russian Federation for Abroad Studies. Now undergoes internship in Queen Mary's University of London, UK.

Sindeev Sergey finished his $\mathrm{PhD}$ course in physiology at Saratov National Research University in 2016. His research interests includes microcirculation dynamics, in vivo experiments, vascular physiology, and pathopysiology. He was awarded by Scholarship of the Russian Federation Government.

Oxana V. Semyachkina-Glushkovskaya is a professor and a head of chair of physiology of human and animals at Saratov State University, Russia. Her research interests are focused on the study of nature of stress and development of stress-related vascular catastrophes in the brain and the application in practical medicine the innovative optical and mathematical technologies for prognosis and treatment of social important stress-induced diseases.

Valery V. Tuchin is a professor and head of Optics and Biophotonics at Saratov National Research State University and several other universities and institutions. His research interests include tissue optics, laser medicine, tissue optical clearing, and nanobiophotonics. He is a fellow of SPIE and OSA, has been awarded Honored Science Worker of the Russia, SPIE Educator Award, FiDiPro (Finland), Chime Bell Prize of Hubei Province (China), and Joseph W. Goodman Book Writing Award (OSA/SPIE). 